\title{
Age-related deficits in efficiency of low-level lateral inhibition
}

\section{Friederike Schlaghecken *, Kulbir S. Birak and Elizabeth A. Maylor}

Department of Psychology, University of Warwick, Coventry, UK

\section{Edited by:}

Nicola De Pisapia, University of

Trento, Italy

Reviewed by:

Petroc Sumner, Cardiff University, UK

Kristen M. Kennedy, University of Texas at Dallas, USA

${ }^{*}$ Correspondence:

Friederike Schlaghecken,

Department of Psychology,

University of Warwick, Coventry,

CVA TAL, UK.

e-mail:f.schlaghecken@

warwick.ac.uk
Background: In a masked prime task using a $0 \mathrm{~ms}$ prime-target inter-stimulus-interval, responses on trials where prime and target match (compatible trials) are usually faster and more accurate than responses where prime and target mismatch (incompatible trials). This positive compatibility effect (PCE) comprises both behavioral benefits on compatible relative to neutral trials, and behavioral costs on incompatible relative to neutral trials. Comparing performance in 2- vs. 4-alternative-response versions of the task indicates that benefits are due to direct priming (i.e., pre-activation) of a motor response, whereas costs reflect an inhibition of the alternative response tendency. The present study employs this paradigm to test the hypothesis that normal aging is associated with a selective deficit in inhibitory function, affecting both low-level motor and higher-level executive control. Experiment and Results: Testing 20 young and 20 older healthy adults, we found that (1) overall, prime-induced benefits were of similar magnitude across age groups, but inhibition-based costs were smaller in older compared to young adults; (2) increasing the number of response alternatives caused the same pattern of unaltered benefits and reduced costs in both age groups; and (3) costs, but not benefits, in the 2-alternative condition were significantly predicted by scores on the digit symbol substitution task (DSST), independently of age and other background variables. Interpretation: Results demonstrate the possibility of isolating an inhibitory component in low-level perceptuo-motor control. Importantly, this component shows an age-related decline in the absence of a corresponding decline of visuo-motor excitability, and appears to be linked to performance on a higher-level processing speed task. We hypothesize that aging might affect the brain's ability to establish precise short-term lateral inhibitory links, and that even in young adults, the efficiency of such links is a significant contributing factor in higher-level cognitive performance.

Keywords: aging, inhibition, motor control, positive compatibility effect, masked priming
Normal aging is typically accompanied by a decline in many cognitive and motor functions. An increasing body of evidence suggests that both a general slowing of processing speed and dysregulation in specific brain regions-particularly the frontal lobes-contribute to this decline (e.g., Bugg et al., 2006). In line with this, an influential model of cognitive aging proposes that reduced inhibitory control, resulting in increased distractibility, is one of the main factors of age-related cognitive decline (Hasher et al., 1999). However, "inhibition" refers not to a specific phenomenon, but to a variety of cognitive functions and processes, each of which might be differently affected by aging (e.g., Kramer et al., 1994; Nigg, 2000; Andres et al., 2008; Collette et al., 2009; Verhaeghen, 2011). Correspondingly, evidence for age-related inhibitory deficits have been observed in some studies, but not in others, depending on the type of inhibition under investigation (see Maylor et al., 2005, for examples). Furthermore, even a seemingly unitary inhibitory process like the suppression of a motor response might show divergent patterns of results, depending on the tasks employed to test it. For instance, investigating response suppression in a Simon and in a priming task, Burle et al. (2005) obtained positively correlated results, suggesting a common underlying mechanism. In contrast, Swick et al. (2011) found that response suppression in a go/nogo and in a stop-signal task relied on only partially overlapping cortical and subcortical structures, suggesting distinct underlying mechanisms.

If even an apparently specific instantiation of inhibition like response suppression might potentially involve various (sub-) processes, each of which might or might not be affected by aging, then it seems advisable to investigate age-related inhibitory deficits using a narrowly defined, highly specific type of inhibition. The present study used the inhibitory component of masked prime-induced motor activation for this purpose.

\section{MOTOR ACTIVATION AND INHIBITION IN THE MASKED PRIME PARADIGM}

The masked prime paradigm is an experimental procedure aimed at investigating low-level, automatic visuo-motor control processes (e.g., Eimer and Schlaghecken, 1998). In this task, participants give a speeded motor response to a simple visual target (e.g., a left-hand key-press to an arrow pointing to the left). Each 
target is preceded by a prime stimulus, which is associated with either the same response as the subsequent target (compatible trial), with a different response (incompatible trial), or is without response assignment (neutral trial). Primes are presented very briefly (e.g., $33 \mathrm{~ms}$ ) and are followed by a patterned backward mask. This makes them unlikely to be perceived consciously (near-threshold or subthreshold presentation), as evidenced by participants' informal verbal reports and by their inability to identify primes with more than chance accuracy (Schlaghecken and Eimer, 1997; Eimer and Schlaghecken, 1998, 2002). Yet these primes can be shown to trigger their corresponding motor activation, thereby influencing responses to the subsequently presented, clearly visible targets.

The priming effects typically present a biphasic pattern (e.g., Aron et al., 2003; Seiss and Praamstra, 2004; Schlaghecken and Maylor, 2005; Sumner and Brandwood, 2008): when prime and target are presented in immediate succession, responses are faster and more accurate on compatible than on incompatible trials (positive compatibility effect, PCE); if target presentation is slightly delayed (by about 100-250 ms), the priming effect reverses (negative compatibility effect, NCE), a pattern indicative of an activation-followed-by-inhibition sequence. Interestingly, it has been found that in healthy older participants, the initial PCE is of approximately the same magnitude as in young participants, whereas the subsequent NCE is much reduced or even absent (e.g., Seiss and Praamstra, 2004; Schlaghecken and Maylor, 2005; Schlaghecken et al., 2011), suggesting that motor inhibition, but not prime-induced motor activation, deteriorates with old age. In contrast to these results, however, Sumner et al. (2007) observed robust NCEs in older participants, suggesting intact motor inhibition. In fact, an analysis of the priming effects of 80 older adults revealed large individual differences in the NCE latency range, with some participants producing "normal" NCEs, but others showing PCEs (Schlaghecken et al., 2011). In that study, no predictor for these differences could be identified. However, it seems likely that the NCE reflects the combined activity of more than one inhibitory process (see, e.g., McBride et al., 2012, for a review), complicating the pattern of deteriorating and spared functions. Thus, it might be interesting to employ a simpler measure of low-level motor inhibition to investigate age-related and individual differences in motor control.

In the typical 2-alternative choice reaction time (RT) task, the PCE comprises behavioral benefits (improved performance on compatible relative to neutral trials) and costs (impaired performance on incompatible relative to neutral trials) of approximately equal magnitude (Aron et al., 2003; Schlaghecken et al., 2006). These effects are generally assumed to reflect activation and inhibition processes in the visuo-motor system (rather than, for instance, processes of perceptual or attentional modulation, e.g., Schlaghecken and Eimer, 2001; Boy and Sumner, 2010). That is, the masked prime is assumed to directly activate its corresponding motor response (e.g., Neumann and Klotz, 1994; Kiesel et al., 2007), resulting in performance benefits when the target requires execution of this same response (compatible trial). Importantly, it is further assumed that the activity level of one visuo-motor channel systematically affects activity in competing channels via reciprocal inhibitory links. Thus, once the prime has activated its corresponding motor response, this increased activity in one channel will cause a corresponding decrease of activity in the alternative response channel, resulting in behavioral costs when the target requires execution of this inhibited response (incompatible trial).

The feasibility of separating direct prime activation and indirect competitor inhibition has been demonstrated in a masked prime experiment where responses had to be given by moving a finger from a central "home" location to one of two or four target locations (Schlaghecken et al., 2006). The logic behind this manipulation was as follows: (1) if masked priming effects are located at the level of motor response codes (rather than either at abstract "left" or "right" codes or at specific muscle commands; see Eimer et al., 2002; Schlaghecken et al., 2009), then they should be unaffected by the type or number of effectors, that is, a single-effector choice RT paradigm should yield the same effects as a two-effector choice RT paradigm; (2) if behavioral benefits on compatible relative to neutral trials reflect a direct ("local") activational effect of the prime, then benefits should remain unaffected by the number of response alternatives; and (3) if behavioral costs on incompatible relative to neutral trials reflect indirect ("global") inhibition of alternative response channels via reciprocal inhibitory links, then costs should decrease with increasing numbers of channels participating in this inhibitory network. Schlaghecken et al. (2006) obtained results exactly in line with these predictions: whereas benefits were virtually identical in the 2- and the 4-alternative condition (9.4 and $9.3 \mathrm{~ms}$ ), costs were substantially reduced (from 14.2 to $4.6 \mathrm{~ms}$ ) with the increased number of response alternatives, confirming that direct activation and reciprocal competitor inhibition processes can be successfully dissociated in the masked prime task, even when different responses are given with the same effector.

\section{RECIPROCAL INHIBITION AND AGING}

Reciprocal inhibitory links appear to be an inherent feature of the perceptuo-motor system, such that activation of one response channel causes a corresponding inhibition of its competing response channel(s) (e.g., Duque et al., 2010; Tandonnet et al., 2011; for a review, see, e.g., Burle et al., 2004). This competitor inhibition is established in a task-dependent manner, not merely through reciprocal inhibition of homologous cortical structures (e.g., Meynier et al., 2009), and might critically depend on pre-supplementary motor area (pre-SMA) circuits (for a review, see Mostofsky and Simmonds, 2008) ${ }^{1}$. Note that this type of inhibition differs from top-down, frontally mediated inhibitory control: it is not the (voluntary) suppression of an incorrect response tendency, but the automatic, feed-forward fine-tuning of the desired response through the suppression of any competing motor activity.

Of particular interest in the present context is the finding that reciprocal inhibition appears to deteriorate with age. This has been demonstrated for both intra-hemispheric (Hortobágyi et al., 2006) and inter-hemispheric (Talelli et al., 2008) task-specific

\footnotetext{
${ }^{1}$ It might be interesting to note that Sumner et al. (2007) found evidence that pre-SMA also plays a role in generating the second, NCE phase of masked prime effects.
} 
inhibition during single responses. Furthermore, van de Laar et al. (2012) have suggested that the same might be true for choice (between-hand) reactions. However, inhibitory links and their age-related changes in the intermediate condition-a choice RT task employing a single-effector-have, to the best of our knowledge, not as yet been investigated. Finding age-related changes in motor inhibition in such a paradigm would demonstrate that aging does indeed affect ad-hoc, task-related perceptuo-motor links, not just anatomical links between antagonistic muscle pairs or homologous motor cortex areas.

The present study, therefore, employed the paradigm used in Schlaghecken et al. (2006), where single finger movements were mapped onto different targets under 2- and 4-alternative response conditions. We compared performance of young and older participants in this paradigm, aiming to replicate and extend the earlier findings by separating the effects of aging on direct motor activation and on reciprocal motor inhibition.

\section{AGING, INHIBITION, AND PROCESSING SPEED}

It is generally agreed that the speed of information processing decreases with increasing age (e.g., Salthouse, 1996, 2004). This is likely due to a combination of reduced nerve conduction velocity (e.g., Stetson et al., 1992) and age-related loss of synaptic connections (e.g., Fjell and Walhovd, 2010), resulting in an overall deterioration of functional neural networks (e.g., Andrews-Hanna et al., 2007; Damoiseaux et al., 2008) and a corresponding deficiency in the formation of neural representations (e.g., Rousselet et al., 2009).

A typical measure of processing speed in many aging studies is the digit symbol substitution task (DSST; see Hoyer et al., 2004). As a subtest of Wechsler intelligence scales, the DSST correlates highly with intelligence and is generally acknowledged to involve higher levels of cognition than simply perceptual speed (e.g., Laux and Lane, 1985; Piccinin and Rabbitt, 1999; Gilmore et al., 2006), possibly including inhibitory processes. Lustig et al. (2006), comparing young and older adults' performance under low- and high-distraction conditions, found that the ability to ignore distracting information is an important factor in DSST and DSST-like measures of processing speed. However, as the authors point out, being able to ignore distractors is not entirely determined by inhibitory control: factors such as visual acuity and gaze control might also play a role. To the best of our knowledge, there has as yet been no study to directly relate processing speed to low-level inhibitory control. Because the present study has been designed to obtain a relatively pure measure of low-level inhibition (behavioral costs on incompatible relative to neutral trials in the 2-alternative version of the masked prime task), it is suitable to address this issue.

\section{METHODS}

\section{PARTICIPANTS}

Twenty young (seven male) and 20 older (nine male) healthy participants completed the experiment (see Table 1 for details). Young participants (aged 18-33) were mostly students at the University of Warwick who took part either for course credit or for payment of $\mathfrak{E} 6$. Older participants (aged 63-81) were members of a volunteer panel who had been recruited through local newspapers and advertisements to join the Warwick Age Study and were paid $\mathfrak{E} 10$ as a contribution toward their travel expenses. All but one young and two older participants were right handed.

\section{BACKGROUND MEASURES}

Crystallized intelligence was assessed by the multiple choice section of the Mill Hill vocabulary test (Raven et al., 1988), in which participants have to select the best synonym for a target word from a set of six alternatives. Speed of information processing was assessed by the DSST from the Wechsler Adult Intelligence Scale-Revised (Wechsler, 1981). Visual acuity was assessed (with glasses if worn) at the beginning of the experiment using the Near Vision Test Card (Schneider, 2002). All participants had normal or corrected-to-normal vision according to self-report. The data in Table 1 show the typical pattern reported in the aging literature (e.g., Baltes and Lindenberger, 1997; Schneider and PichoraFuller, 2000) of higher processing speed and visual acuity, but lower crystallized intelligence, in young compared with older adults. Moreover, the DSST means correspond almost exactly with those reported for 20 - vs. 70 -year-olds (70 vs. 49 , respectively) in a meta-analysis of 141 studies by Hoyer et al. (2004), again suggesting that the present samples are representative of their age group.

\section{STIMULI AND APPARATUS}

Double arrows pointing left, right, up, or down served as primes and targets, and a double plus sign served as an additional neutral prime (see Figure 1). Stimuli subtended a visual angle of $1.2^{\circ} \times 0.6^{\circ}$ at a viewing distance of approximately $1 \mathrm{~m}$. Masks were constructed from a virtual $8 \times 6$ grid $\left(2.3^{\circ} \times 1.4^{\circ}\right)$, randomly filled with overlapping horizontal, vertical, and oblique

Table 1 | Background details (means and standard deviations) of young and older participants, and results of comparisons between age groups.

\begin{tabular}{llll}
\hline Variable & Young & Older & \\
\hline & M (SD) & M (SD) & Comparison \\
\hline Age (years) & $22.3(4.3)$ & $70.3(5.3)$ & - \\
Health $^{a}$ & $4.7(0.5)$ & $4.1(0.6)$ & $t_{(34)}=3.23, p<0.01$ \\
Vocabulary $^{b}$ & $18.5(4.0)$ & $23.2(2.8)$ & $t_{(36)}=-4.23, p<0.001$ \\
Speed $^{c}$ & $70.1(14.9)$ & $50.8(10.4)$ & $t_{(36)}=4.67, p<0.001$ \\
Visual acuity $^{d}$ & $7.0(1.1)$ & $5.1(1.3)$ & $t_{(38)}=5.06, p<0.001$ \\
\hline
\end{tabular}

a Self-rated health on a 5-point scale (very poor; poor; fair; good; very good); data missing for two young and two older participants.

${ }^{b}$ Vocabulary from the multiple choice section of the Mill Hill vocabulary test (Raven et al., 1988); maximum score = 33; data missing for two young participants.

cProcessing speed based on the Digit Symbol Substitution task (Wechsler, 1981); data missing for two young participants.

'Visual acuity as measured by the number of lines read correctly from the Near Vision Test Card (Schneider, 2002) viewed at a distance of 16 inches whilst wearing corrective glasses, with scores ranging from 1 (16/160-lowest acuity) to 9 (16/16 —highest acuity). 

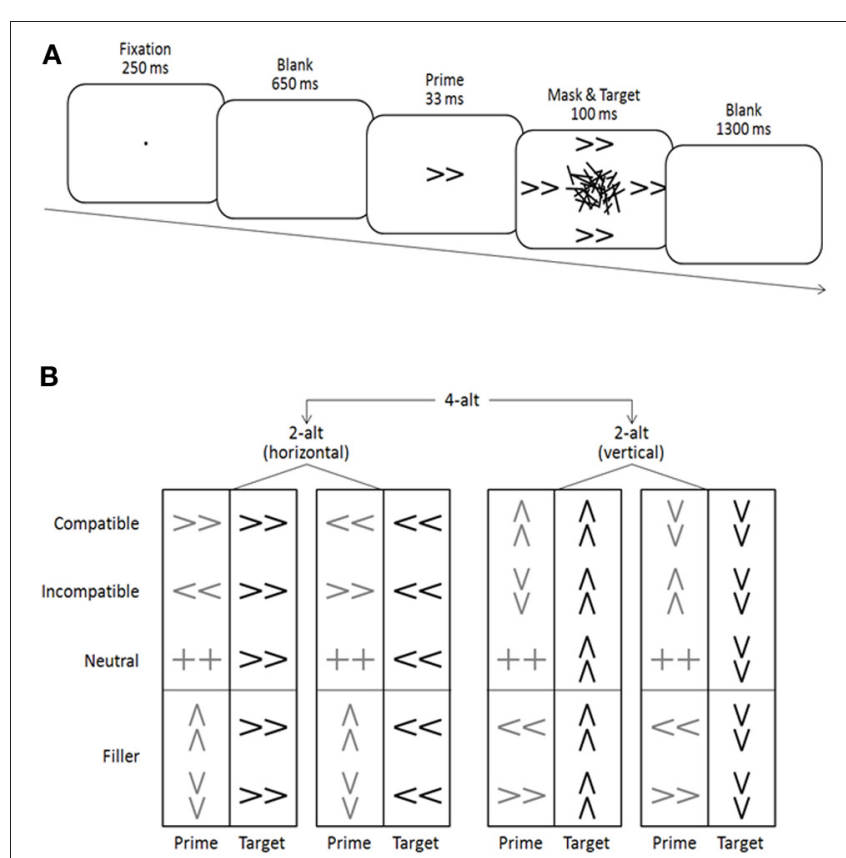

FIGURE 1 | (A) Schematic illustration of a (compatible) trial. (B) List of all possible trials in the two 2-alternative (2-alt) choice tasks and the combined 4-alternative (4-alt) choice task.

lines of different lengths $\left(0.1^{\circ}-1.0^{\circ}\right.$; width $\left.0.2^{\circ}\right)$. A new mask was constructed on each trial to avoid perceptual learning of the mask and correspondingly increased prime identification (see Schubö et al., 2001; Schlaghecken et al., 2008). Stimuli were presented in black on a white background on a $17^{\prime \prime}$ computer screen. A fixation $\operatorname{dot}\left(0.1^{\circ} \times 0.1^{\circ}\right.$ visual angle $)$, primes and masks appeared in the center of the screen, whereas targets appeared $2.0^{\circ}$ above, below, to the left and to the right of the center (i.e., just beyond the area occupied by the mask-see Figure 1A). Participants were seated in a comfortable chair in a dimly lit, sound attenuated chamber approximately $1 \mathrm{~m}$ in front of a computer screen. They pressed the " 2 ," " 4 ," " 6 ," or " 8 " key on the number pad of a standard keyboard in response to the corresponding target direction (down, left, right, and up, respectively).

\section{PROCEDURE}

In experimental sessions lasting up to $1 \mathrm{~h}$, participants first carried out eight blocks of the 2-alternative choice RT task (2-alt), then eight blocks of the 4-alternative choice RT task (4-alt). Immediately after the experiment, background cognitive measures were collected from young participants (this information was already available for older participants from an earlier experiment).

A typical trial sequence is depicted in Figure 1A. Trials started with a fixation cross presented for $250 \mathrm{~ms}$, followed by a $650 \mathrm{~ms}$ blank screen. A prime was then presented for $33 \mathrm{~ms}$, replaced immediately by a 100 ms mask surrounded by four identical targets. The inter-trial interval between target offset and the next fixation dot was $1300 \mathrm{~ms}$. On compatible trials, prime and target arrows pointed in the same direction, on incompatible trials, they pointed in opposite directions (e.g., left-pointing prime, right-pointing target), and on neutral trials, the prime was a double-plus sign not associated with any response. Trials where prime direction was orthogonal to target direction (e.g., leftpointing prime, upward-pointing target) were considered as filler trials, and were not part of the main analysis.

Participants were instructed to maintain central eye fixation, and to respond as quickly and accurately as possible to the direction of the target arrows by moving the index finger of the right hand from its resting position on the central " 5 " key in order to press the key indicated by the target arrow direction (down to " 2 ," left to " 4 ," etc.). Participants initially practiced this under the supervision of the experimenter.

The two parts of the experiment differed only regarding the number of different targets presented within a given block (two vs. four), and were identical in all other respects of stimulus presentation (see Figure 1B). Specifically, all five different primes-left, right, up, down, neutral-were presented in each block. However, in a 2-alt block, all targets came from only one spatial dimension (i.e., either all horizontal [left/right], or all vertical [up/down]), whereas in a 4 -alt block, targets from both spatial dimensions were mixed. Thus, in effect, each 4 -alt block was the combination of one 2-alt horizontal and one 2-alt vertical block.

Responses were expected to be substantially slower in the 4-alt than in the 2-alt task (Hick, 1952; Hyman, 1953). In order to minimize these RT differences, the eight 2-alt blocks (60 trials each) were presented first. Half of the participants started with a series of four 2-alt horizontal blocks followed by a series of four 2-alt vertical blocks (see Figure 1B); for the other half, this sequence was reversed. Forty practice trials were given at the beginning of each series. At the end of the first part, participants were encouraged to leave the experimental room for a short rest period. The second part (again starting with a 40 -trial practice phase) consisted of eight 4 -alt blocks (60 trials each). An overview of all 20 possible prime-target combinations ( 5 prime $\times 4$ targets) and their distribution across conditions is provided in Figure 1B. Within each block, all prime-target combinations were presented randomly and with equal probability, resulting in 20\% compatible, $20 \%$ neutral, and $20 \%$ incompatible trials, plus $40 \%$ filler trials ${ }^{2}$.

\section{DATA ANALYSES}

Repeated measures analyses of variance (ANOVAs) were performed on mean correct RTs, with the between-subject factor of age (young vs. older), and the within-subject factors of task (2-alt vs. 4-alt) and trial type (compatible vs. neutral vs. incompatible). In all analyses, Greenhouse-Geisser correction to the

\footnotetext{
${ }^{2}$ The large number of filler trials is an unfortunate side effect of two constraints: (a) primes had to be non-predictive of the upcoming target, because even with subliminal presentation, priming effects are modulated by the primes' predictiveness (see Klapp, 2007). This means that in the 4-alt blocks, a left-pointing prime, for instance, had to be followed with equal probability by a left-, a right-, an up-, and a down-pointing target; and (b) the relevant manipulation in this experiment was the number of possible targets within a block (two vs. four), so consequently, all other factors-particularly the relative frequency of different prime-target combinations-were kept identical between conditions.
} 
degrees of freedom was applied where appropriate, and corrected $p$-values are reported. Because of the large RT difference between age groups (see below), priming effects were calculated not as RT differences, but as RT ratios (benefits = neutral RTs : compatible RTs; costs = incompatible RTs : neutral RTs). ANOVAs were performed on priming effects with the between-subject factor of age group, and the within-subject factors of task and type (benefit vs. cost). Follow-up analyses were conducted in the form of $t$-tests.

Additionally, RTs on filler trials relative to compatible and incompatible RTs were analyzed. Note that in the 2-alt task, primes orthogonally oriented relative to the target have the same general stimulus characteristics as compatible and incompatible primes, but have no explicit response assignment. Therefore, they can be expected to produce RTs intermediate between compatible and incompatible RTs (Wilson et al., 2010). In the 4-alt task, in contrast, these filler primes do have a response assignment, and therefore should affect the visuo-motor system in the same ways as any other incompatible prime. Analyses were conducted using paired $t$-tests, separately for the 2-alt and the 4-alt task and for both age groups. Overall error rates were extremely low (1-3\% on average) and thus were not further analyzed.

\section{RESULTS}

As can be seen from Figure 2, older adults were around $250 \mathrm{~ms}$ slower than young adults, $\left[F_{(1,38)}=68.18, p<0.001\right]$. Although RTs were numerically longer with four than with two response alternatives, this difference was not significant, nor did it interact with age, both $F s<1.7$, both $p s>0.20$. There was a highly significant effect of trial type, as RTs increased from compatible to neutral to incompatible trials, $\left[F_{(1,38)}=136.67, p<0.001\right]$. This priming effect interacted with task (larger priming effects in the 2 -alt than in the 4 -alt task, $\left.\left[F_{(1,38)}=18.76, p<0.001\right]\right)$, and with age (young adults showing larger priming effects than older adults, $\left.\left[F_{(1,38)}=3.85, p=0.026\right]\right)$, but there was no three-way interaction between these factors, $F<1$.

\section{FILLER-TRIAL ANALYSES}

These confirmed our predictions about the impact of a response assignment on priming effects: in the 2-alt task, filler-trial RTs were significantly longer than compatible RTs, and significantly shorter than incompatible RTs, for both young and older adults, all $t \mathrm{~s}_{(19)}>3.76$, all $p \mathrm{~s}<0.002$. Furthermore, the two RT differences (filler RT minus compatible RT, and incompatible RT minus filler RT) were of approximately the same magnitude in both age groups, both $t s<1.16$, both $p s>0.25$. Filler RTs in the 4 -alt task were also significantly longer than compatible RTs for both young and older adults, both $t$ s $>6.90$, both $p s<0.001$, but in contrast to the 2-alt task, they did not differ significantly from incompatible RTs, both $t$ s $<1.3$, both $p s>0.22$.

\section{BEHAVIORAL BENEFITS AND COSTS}

These are depicted in Figure 3, expressed as ratios of compatible (benefits) and incompatible (costs) to neutral trials (rescaled such that 0 rather than 1 indicates equality of antecedent and consequent). Both types of effects were smaller in older than in young adults, $\left[F_{(1,38)}=17.17, p<0.001\right]$. However, this age difference was far more pronounced for costs than for benefits,

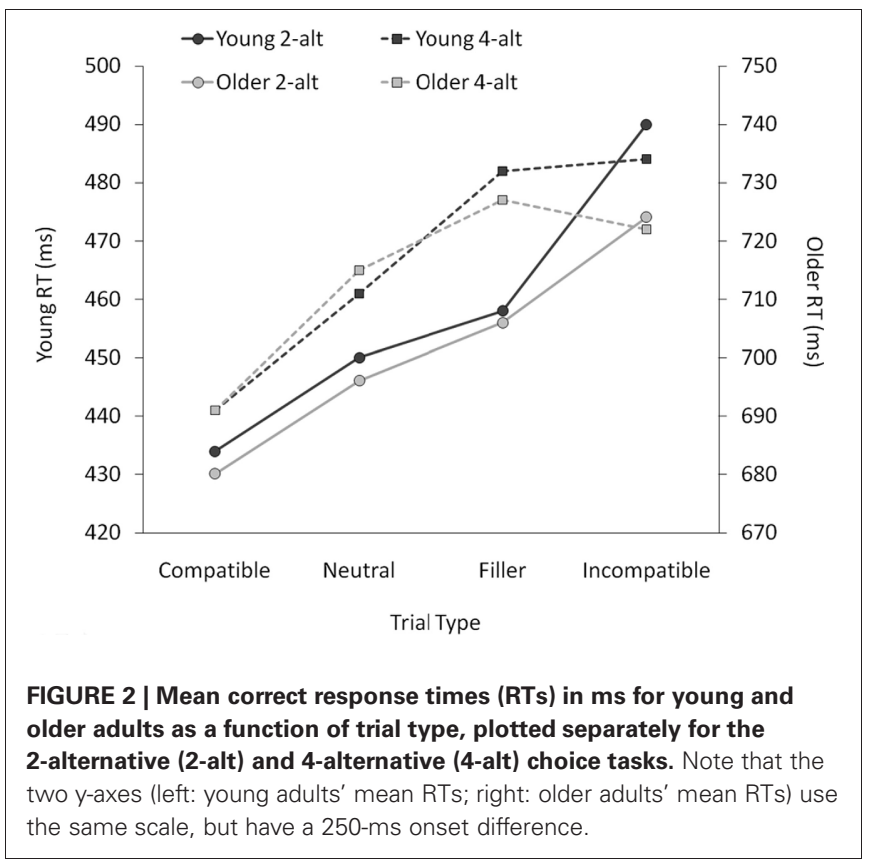

as indicated by a significant Age $\times$ Type interaction, $\left[F_{(1,38)}=\right.$ $10.38, p<0.001]$. Follow-up $t$-tests confirmed that whereas benefits did not differ significantly between young and older adults, both $t$ s $<1.7$, both $p s>0.11$, costs did, both $t$ s $>3.66$, both ps $<0.001$. Furthermore, overall costs were larger than overall benefits, $\left[F_{(1,38)}=23.86, p<0.01\right]$, and priming effects were overall larger in the 2 -alt than in the 4 -alt task, $\left[F_{(1,38)}=5.49\right.$, $p=0.024]$. These two effects interacted significantly, as the costbenefit difference was much larger in the 2-alt than in the 4 -alt task, $\left[F_{(1,38)}=20.74, p<0.001\right]$. Specifically, whereas benefits remained largely unaffected by the number of response alternatives (2-alt vs. 4 -alt: $t_{(19)}<1.6, p>.13$, for both young and older adults), costs were substantially reduced with larger numbers of response alternatives in both young and older adults, both ts $>3.7$, both $p s<0.001^{3}$. Neither the main effect of task, nor the Type $\times$ Task interaction, interacted with age, both $F$ s $<1.9$, both $p s>0.18$.

Next, we conducted multiple regression analyses to examine whether the four priming effects (behavioral benefits and costs in the 2-alt and 4-alt tasks) were independently predicted by any of the background measures (age, visual acuity, vocabulary, and DSST score). The overall regression models were not significant for either 2-alt benefits, adjusted $R^{2}=-0.041 ; F<1$, or 4-alt benefits, adjusted $R^{2}=0.057 ;\left[F_{(4,33)}=1.56, p>0.2\right]$. However, for 2-alt costs, the overall model was highly significant, adjusted $R^{2}=0.573 ;\left[F_{(4,33)}=13.44, p<0.001\right]$, with DSST alone making an independent contribution to the variance, $t=$ 4.59, $p<0.001$, such that faster processing speed (higher DSST

\footnotetext{
${ }^{3}$ A block-wise analysis of costs showed no gradual decrease in costs over time: costs remained constant throughout the first half of the experiment (2-alt task), then dropped sharply and stayed at the new lower level throughout the second half (4-alt task), thus confirming that the difference in cost magnitude between 2 - and 4 -alt tasks was not simply due to fatigue.
} 


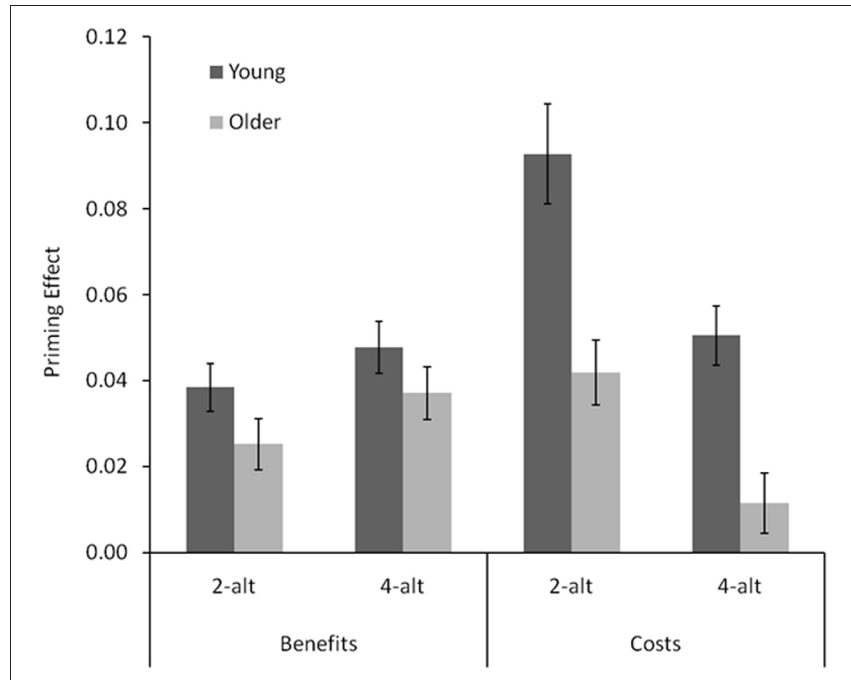

FIGURE 3 | Benefits (neutral RT : compatible RT ratio minus 1) and costs (incompatible RT : neutral RT ratio minus 1) for young and older adults in the 2-alt and 4-alt choice tasks. Error bars represent standard errors.

score) was associated with greater reciprocal inhibition (larger behavioral costs; see Figure 4). The overall model for 4-alt costs was also significant, adjusted $R^{2}=0.288$; $\left[F_{(4,33)}=4.74, p<\right.$ 0.005 ], but no measure made a significant independent contribution to the variance (DSST being closest at $t=1.61, p=0.12$ ). In other words, with age, visual acuity, and vocabulary already taken into account, DSST significantly predicted behavioral costs in the 2-alt task.

\section{DISCUSSION}

Comparing performance in a masked prime task with two and four response alternatives (2-alt vs. 4 -alt), the present experiment aimed to confirm that prime-induced motor activation and inhibition processes can be dissociated, and to examine the effect of aging on these separate components. Results replicated the findings of Schlaghecken et al. (2006; Exp. 1): (1) relative to neutral trials, masked primes triggered behavioral benefits on compatible and costs on incompatible trials (PCE); (2) in the 2-alt task, costs were noticeably larger than benefits, possibly because with the stimuli employed in the present experiment, unspecific motor activation triggered by neutral primes (plus signs) is stronger than unspecific activation triggered by arrow primes, leading to a shortening of RTs on neutral-prime trials relative to arrow-prime trials (e.g., Hasbroucq et al., 1999); (3) overall, the PCE was smaller in the 4-alt than in the 2-alt task; and (4) this effect was entirely driven by a reduction of costs with increasing number of response alternatives, whereas the magnitude of benefits was unaffected by this manipulation. The results thus confirm the notion that whereas (compatible-trial) priming benefits measure direct prime activation, (incompatible-trial) priming costs measure the separate and dissociable process of indirect reciprocal competitor inhibition. Importantly, these patterns were observed for both young and older adults, despite the overall differences in response speed (RTs) and competitor inhibition (behavioral costs).

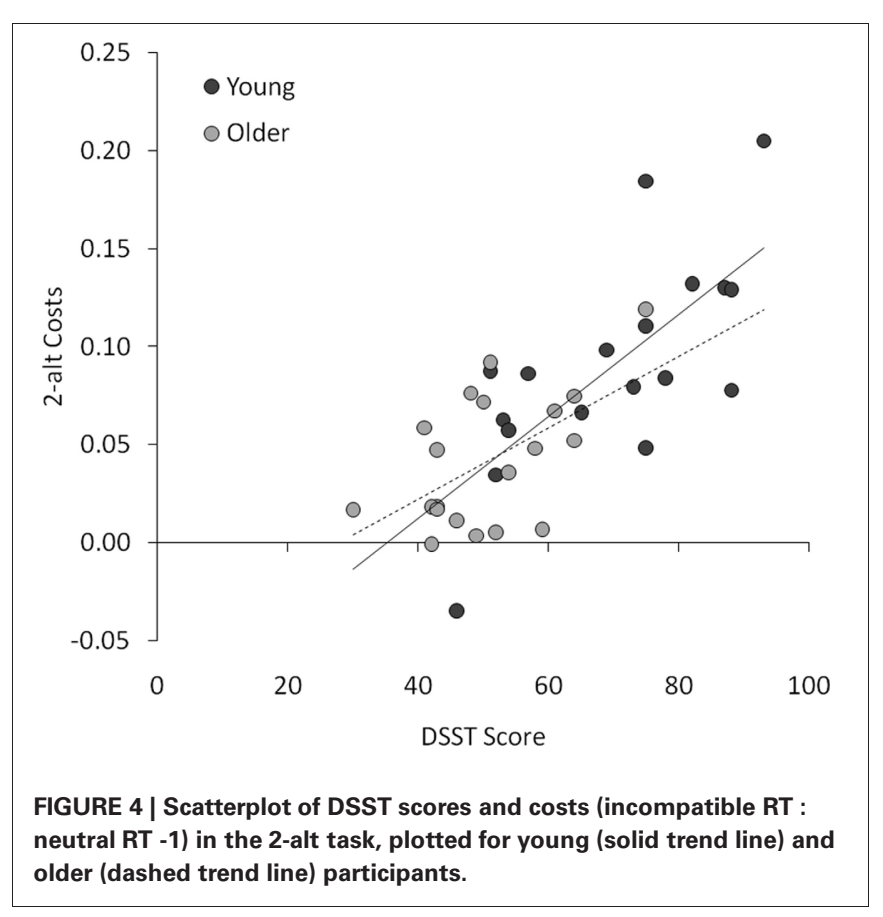

Reciprocal competitor inhibition is assumed to differ from top-down inhibitory control in that it is low-level, automatic, and feed-forward, aimed at fine-tuning the ongoing response execution through the prevention of incorrect response activations (rather than through the suppression of an already activated incorrect response; e.g., Burle et al., 2004; Duque et al., 2010; Tandonnet et al., 2011). Yet as demonstrated by Meynier et al. (2009), this type of inhibition is not hard-wired, but is established in a flexible, task-dependent way. The aim of the present study was to investigate (a) the effect of aging on this type of inhibition, and (b) its possible relationship to speed of processing. Results clearly demonstrated that in the masked prime task, aging selectively affects reciprocal competitor inhibition, not prime-induced motor activation: behavioral costs were much smaller in older than in young adults, whereas behavioral benefits were of similar magnitude. This pattern is in line with the notion that unlike top-down, cue-related activation (e.g., Sterr and Dean, 2008), low-level, prime-induced motor activation remains stable across the adult lifespan (e.g., Schlaghecken and Maylor, 2005), whereas reciprocal inhibitory links in the motor response system deteriorate with advanced age (e.g., Hortobágyi et al., 2006).

It is probably worth noting that in the present experiment, age-related reductions in inhibitory efficiency are reflected in smaller interference effects in older compared to young adults, whereas typically, they are reflected in larger effects. For instance, in classical response conflict paradigms like the Simon or the Eriksen flanker task, where on incompatible trials an automatically activated incorrect motor response interferes with the execution of the correct response, older participants typically show larger interference effects than young participants, suggesting that their inhibitory control mechanisms are less efficient in overcoming 
the incorrect response tendency (e.g., van der Lubbe and Verleger, 2002). Those tasks, however, differ from the present one in a crucial aspect: they measure reactive or top-down inhibition of a response that has already been activated. Thus, the less effective the inhibition, the stronger the interference from this inappropriate response. The present task, in contrast, measures the reciprocal inhibition of a response that has not yet been activated. Consequently, the less effective this inhibition, the less this response channel will be deactivated below baseline levels, and the less the execution of this response will be delayed. One might argue, of course, that reactive inhibition of an incorrectly activated response does play a role in the present task as well. However, one needs to keep in mind that because primes were masked, and therefore only of limited accessibility to conscious or high-level control processes, it is likely that frontally mediated top-down inhibition of the incorrect (incompatible) motor activation will have played only a relatively minor role in the current task (e.g., Dehaene et al., 2003; Schlaghecken et al., 2011).

This is not to say that frontal areas are not involved in non-consciously triggered control processes at all. Specifically, the supplementary motor areas (SMA/pre-SMA) have been shown to be directly related to priming effects in the masked prime task (Sumner et al., 2007; van Gaal et al., 2011), and even the anterior cingulate cortex-one of the central structures in high-level cognitive control-has been shown to respond to stimulus irregularities (Ursu et al., 2009) and response errors (Nieuwenhuis et al., 2001) of which participants are not consciously aware. It seems tempting to speculate whether these apparent separate forms of inhibitory control are merely increasingly complex or wide-spread instantiations of the same underlying basic process of reciprocal inhibition. Future research will undoubtedly explore this issue in more detail, specifically with regard to inhibitory dysregulation in psychological and neurological disorders and in normal aging.

The present study provides one step in this direction, as it demonstrated that the strengths of reciprocal inhibitory links might be related to the general speed of information processing. Independent of age, visual acuity, and crystallized intelligence, processing speed (as measured by DSST scores) positively predicted competitor inhibition (as measured by the magnitude of behavioral costs in the 2 -alt masked prime task). To the best of our knowledge, this is the first time that direct evidence has been provided that performance in the DSST is associated with low-level inhibitory control. Performance in processing speed tasks has previously been linked to the ability to ignore distracting information (Lustig et al., 2006), an ability strongly associated with prefrontal lobe functions (e.g., Everling et al., 2002). However, the present results-while generally supporting the notion of a relationship between DSST performance and inhibition-suggest that this relationship might be mediated by more basic processes than top-down inhibitory control. One possible interpretation is that more efficient reciprocal inhibition of competing response alternatives allows for generally faster response execution (recall that mean RTs in the priming task were approximately $250 \mathrm{~ms}$ shorter in young than in older participants), resulting in the ability to produce more responses within a given amount of time. This interpretation, however, seems somewhat simplistic, and does not fit well with the present data: if faster response execution due to reciprocal inhibition accounts for improved DSST performance, then DSST scores should have been negatively correlated with RTs, particularly on compatible trials, where the "foregrounded" response has to be executed. This was not the case (all $r \mathrm{~s}<0.40$, all $p s>0.08)$, suggesting that DSST scores are not merely a function of efficient motor execution. Alternatively, one might assume that the same neural mechanism that instantiates reciprocal inhibition in the motor system produces the attentional focus needed in the DSST and similar processing speed tasks. Again, future research will have to investigate this issue more directly.

\section{ACKNOWLEDGMENTS}

This research was supported by a grant to Elizabeth A. Maylor and Friederike Schlaghecken from the Economic and Social Research Council of Great Britain (RES-000-22-2687).

\section{REFERENCES}

Andres, P., Guerrini, C., Phillips, L. H., and Perfect, T. J. (2008). Differential effects of aging on executive and automatic inhibition. Dev. Neuropsychol. 33, 101-123.

Andrews-Hanna, J. R., Snyder, A. Z., Vincent, J. L., Lustig, C., Head, D., Raichle, M. E., and Buckner, R. L. (2007). Disruption of large-scale brain systems in advanced aging. Neuron 56, 924-935.

Aron, A., Schlaghecken, F., Fletcher, P., Bullmore, E., Eimer, M., Barker, R., Sahakian, B., and Robbins, T. (2003). Inhibition of subliminally primed responses is mediated by the caudate and thalamus: evidence from fMRI and Huntington's disease. Brain 126, 713-723.
Baltes, P. B., and Lindenberger, U. (1997). Emergence of a powerful connection between sensory and cognitive functions across the adult life span: a new window to the study of cognitive aging? Psychol. Aging $12,12-21$.

Boy, F., and Sumner, P. (2010). Tight coupling between positive and reversed priming in the masked prime paradigm. J. Exp. Psychol. Hum. Percept. Perform. 36, 892-905.

Bugg, J. M., Zook, N. A., DeLosh, E. L., Davalos, D. B., and Davis, H. P. (2006). Age differences in fluid intelligence: contributions of general slowing and frontal decline. Brain Cogn. 62, 9-12.

Burle, B., van den Wildenberg, W. P. M., and Ridderinkhof, K. R. (2005).
Dynamics of facilitation and interference in cue-priming and Simon tasks. Eur. J. Cogn. Psychol. 17, 619-641.

Burle, B., Vidal, F., Tandonnet, C., and Hasbroucq, T. (2004). Physiological evidence for response inhibition in choice reaction time tasks. Brain Cogn. 56, 153-164.

Collette, F., Germain, S., Hogge, M., and van der Linden, M. (2009). Inhibitory control of memory in normal ageing: dissociation between impaired intentional and preserved unintentional processes. Memory 17, 104-122.

Damoiseaux, J. S., Beckmann, C. F., Arigita, E. J., Barkhof, F., Scheltens, P., Stam, C. J., Smith, S. M., and Rombouts, S. A. (2008). Reduced resting-state brain activity in the "default network" in normal aging. Cereb. Cortex 18, 1856-1864.

Dehaene, S., Artiges, E., Naccache, L., Martelli, C., Viard, A., Schurhoff, F., Recasens, C., Martinot, M. L. P., Leboyer, M., and Martinot, J. L. (2003). Conscious and subliminal conflicts in normal subjects and patients with schizophrenia: the role of the anterior cingulate. Proc. Natl. Acad. Sci. U.S.A. 100, 13722-13727.

Duque, J., Lew, D., Mazzocchio, R. Olivier, E., and Ivry, R. B. (2010). Evidence for two concurrent inhibitory mechanisms during response preparation. J. Neurosci. 30, 3793-3802.

Eimer, M., and Schlaghecken, F. (1998). Effects of masked stimuli on motor activation: behavioral and electrophysiological evidence. J. Exp. 
Psychol. Hum. Percept. Perform. 24, 1737-1747.

Eimer, M., and Schlaghecken, F. (2002). Links between conscious awareness and response inhibition: evidence from masked priming. Psychon. Bull. Rev. 9, 514-520.

Eimer, M., Schubö, A., and Schlaghecken, F. (2002). The locus of inhibition in the masked priming of response alternatives. J. Mot. Behav. 34, 3-10.

Everling, S., Tinsley, C. J., Gaffan, D., and Duncan, J. (2002). Filtering of neural signals by focused attention in the monkey prefrontal cortex. Nat. Neurosci. 5, 671-676.

Fjell, A. M., and Walhovd, K. B. (2010). Structural brain changes in aging: courses, causes, and cognitive consequences. Rev. Neurosci. 21, 187-221.

Gilmore, G. C., Spinks, R. A., and Thomas, C. W. (2006). Age effects in coding tasks: componential analysis and test of the sensory deficit hypothesis. Psychol. Aging 21, 7-18.

Hasbroucq, T., Osman, A., Possamal, C.-A., Burle, B., Carron, S., Dépy, D., Latour, S., and Mouret, I. (1999). Cortico-spinal inhibition reflects time but not event preparation: neural mechanisms of preparation dissociated by transcranial magnetic stimulation. Acta Psychol. 101, 243-266.

Hasher, L., Zacks, R. T., and May, C. P. (1999). "Inhibitory control, circadian arousal, and age," in Attention and Performance XVII, eds D. Gopher, and A. Koriat (Cambridge, MA: MIT Press), 653-675.

Hick, W. E. (1952). On the rate of gain of information. Q. J. Exp. Psychol. 4, $11-26$.

Hortobágyi, T., del Olmo, M. F., and Rothwell, J. C. (2006). Age reduces cortical reciprocal inhibition in humans. Exp. Brain Res. 171, 322-329.

Hoyer, W. J., Stawski, R. S., Wasylyshyn, C., and Verhaeghen, P. (2004). Adult age and digit symbol substitution performance: a meta-analysis. Psychol. Aging 19, 211-214.

Hyman, R. (1953). Stimulus information as a determinant of reaction time. J. Exp. Psychol. 45, 188-196.

Kiesel, A., Kunde, W., and Hoffmann, J. (2007). Mechanisms of subliminal response priming. $A d v . \operatorname{Cog} n$. Psychol. 3, 307-315.

Klapp, S. T. (2007). Nonconscious control mimics a purposeful strategy: strength of Stroop-like interference is automatically modulated by proportion of compatible trials. J. Exp. Psychol. Hum. Percept. Perform. 33, 1366-1376.
Kramer, A. F., Humphrey, D. G., Larish, J. F., Logan, G. D., and Strayer, D. L. (1994). Aging and inhibition: beyond a unitary view of inhibitory processing in attention. Psychol. Aging 9, 491-512.

Laux, L. F., and Lane, D. M. (1985). Information processing components of substitution test performance. Intelligence 9, 111-136.

Lustig, C., Hasher, L., and Tonev, S. T. (2006). Distraction as a determinant of processing speed. Psychon. Bull. Rev. 13, 619-625.

Maylor, E. A., Schlaghecken, F., and Watson, D. G. (2005). "Aging and inhibitory processes in memory, attentional, and motor tasks," in Cognitive limitations in Aging and Psychopathology, eds R. W. Engle, G. Sedek, U. von Hecker, and D. N. McIntosh (New York, NY: Cambridge, University Press), 313-345.

McBride, J., Boy, F., Husain, M., and Sumner, P. (2012). Automatic motor activation in the executive control of action. Front. Hum. Neurosci. 6:82. doi: 10.3389/ fnhum.2012.00082

Meynier, C., Burle, B., Possamai, C.-A., Vidal, F., and Hasbroucq, T. (2009). Neural inhibition and interhemispheric connections in two-choice reaction time: a Laplacian ERP study. Psychophysiology 46, 726-730.

Mostofsky, S. H., and Simmonds, D. J. (2008). Response inhibition and response selection: two sides of the same coin. J. Cogn. Neurosci. 20, 751-761.

Neumann, O., and Klotz, W. (1994). Motor responses to nonreportable, masked stimuli - where is the limit of direct parameter specification? Atten. Perform. 15, 123-150.

Nieuwenhuis, S., Ridderinkhof, K. R., Blom, J., Band, G. P. H., and Kok, A. (2001). Error-related brain potentials are differentially related to awareness of response errors: evidence from an antisaccade task. Psychophysiology 38, 752-760.

Nigg, J. T. (2000). On inhibition/disinhibition in developmental psychopathology: views from cognitive and personality psychology and a working inhibition taxonomy. Psychol. Bull. 126, 220-246.

Piccinin, A. M., and Rabbitt, P. M. A. (1999). Contribution of cognitive abilities to performance and improvement on a substitution coding task. Psychol. Aging 14, 539-551.

Raven, J. C., Raven, J., and Court, J. H. (1988). The Mill Hill Vocabulary Scale. London: H. K. Lewis Co. Ltd.
Rousselet, G. A., Husk, J. S., Pernet, C. R., Gaspar, C. M., Bennett, P. J., and Sekuler, A. B. (2009). Agerelated delay in information accrual for faces: evidence from a parametric, single-trial EEG approach. $B M C$ Neurosci. 10, 114.

Salthouse, T. A. (1996). The processingspeed theory of adult age differences in cognition. Psychol. Rev. 103 403-428.

Salthouse, T. A. (2004). What and when of cognitive aging. Curr. Dir. Psychol. Sci. 13, 140-144.

Schlaghecken, F., Birak, K. S., and Maylor, E. A. (2011). Age-related deficits in low-level inhibitory motor control. Psychol. Aging 26, 905-918.

Schlaghecken, F., Blagrove, E., and Maylor, E. A. (2008). No difference between conscious and nonconscious visuomotor control: evidence from perceptual learning in the masked prime task. Conscious. Cogn. 17, 84-93.

Schlaghecken, F., Bowman, H., and Eimer, M. (2006). Dissociating local and global levels of perceptuomotor control in masked priming. J. Exp. Psychol. Hum. Percept. Perform. 32, 618-632.

Schlaghecken, F., and Eimer, M. (1997). The influence of subliminally presented primes on response preparation. Spr. Kogn. 16, 166-175.

Schlaghecken, F., and Eimer, M. (2001). Partial response activation to masked primes is not dependent on response readiness. Percept. Mot. Skills 92, 208-222.

Schlaghecken, F., Klapp, S. T., and Maylor, E. A. (2009). Either or neither, but not both: locating the effects of masked primes. Proc. Biol. Sci. 276, 515-521.

Schlaghecken, F., and Maylor, E. A (2005). Motor control in old age: evidence of impaired low-level inhibition. J. Gerontol. B Psychol. Sci. Soc. Sci. 60, P158-P161.

Schlaghecken, F., Refaat, M., and Maylor, E. A. (2011). Multiple systems for cognitive control: evidence from a hybrid prime-Simon task. J. Exp. Psychol. Hum. Percept. Perform. 37, 1542-1553.

Schneider, B. A., and Pichora-Fuller, M. K. (2000). "Implications of perceptual deterioration for cognitive aging research," in The Handbook of Aging and Cognition, 2nd edn. eds F. I. M. Craik and T. A. Salthouse (Mahwah, NJ: Erlbaum), 155-219.

Schneider, J. (2002). Near Vision Test Card. Retrieved November 16, 2007, from http://www.isee.org/block_letter_eye_chart.pdf
Schubö, A., Schlaghecken, F., and Meinecke, C. (2001). Learning to ignore the mask in texture segmentation tasks. J. Exp. Psychol. Hum. Percept. Perform. 27, 919-931.

Seiss, E., and Praamstra, P. (2004). The basal ganglia and inhibitory mechanisms in response selection: evidence from subliminal priming of motor responses in Parkinson's disease. Brain 127, 330-339.

Sterr, A., and Dean, P. (2008). Neural correlates of movement preparation in healthy ageing. Eur. J. Neurosci. 27, 254-260.

Stetson, D. S., Albers, J. W., Silverstein, B. A., and Wolfe, R. A. (1992). Effects of age, sex, and anthropometric factors on nerve conduc tion measures. Muscle Nerve 15, 1095-1104.

Sumner, P., and Brandwood, T. (2008). Oscillations in motor priming: positive rebound follows the inhibitory phase in the masked prime paradigm. J. Mot. Behav. 40, 484-489.

Sumner, P., Nachev, P., Morris, P., Peters, A. M., Jackson, S. R., Kennard, C., and Husain, M. (2007). Human medial frontal cortex mediates unconscious inhibition of voluntary action. Neuron 54, 697-711.

Swick, D., Ashley, V., and Turken, U. (2011). Are the neural correlates of stopping and not going identical? Quantitative meta-analysis of two response inhibition tasks. Neuroimage 56 1655-1665.

Talelli, P., Waddingham, W., Ewas, A., Rothwell, J. C., and Ward, N. S (2008). The effect of age on taskrelated modulation of interhemispheric balance. Exp. Brain Res. 186, 59-66.

Tandonnet, C., Garry, M. I., and Summers, J. J. (2011). Selective suppression of the incorrect response implementation in choice behavior assessed by transcranial magnetic stimulation. Psychophysiology 48, 462-469.

Ursu, S., Clark, K. A., Aizenstein, H. J., Stenger, V. A., and Carter, C. (2009). Conflict-related activity in the caudal anterior cingulate cortex in the absence of awareness. Biol. Psychol. 80, 279-286.

van de Laar, M., van den Wildenberg, W. P. M., van Boxtel, G. J. M., Huizenga, H. M., and van der Molen, M. W. (2012). Lifespan changes in motor activation and inhibition during choice reactions: 
a Laplacian ERP study. Biol. Psychol. 89, 323-334.

van der Lubbe, R. H. J., and Verleger, R. (2002). Aging and the Simon task. Psychophysiology 39, 100-110.

van Gaal, S., Scholte, H. S., Lamme, V. A. F., Fahrenfort, J. J., and Ridderinkhof, K. R. (2011). PreSMA gray-matter density predicts individual differences in action selection in the face of conscious and unconscious response conflict. J. Cogn. Neurosci. 23, 382-390.

Verhaeghen, P. (2011). Aging and executive control: reports of a demise greatly exaggerated. Curr. Dir. Psychol. Sci. 20, 174-180.

Wechsler, D. (1981). Manual for the Wechsler Adult Intelligence Scale-Revised. New York, NY: Psychological Corporation.

Wilson, A. D., Tresilian, J. R., and Schlaghecken, F. (2010). Continuous priming effects on discrete response choices. Brain Cogn. 74, 152-159.

Conflict of Interest Statement: The authors declare that the research was conducted in the absence of any commercial or financial relationships that could be construed as a potential conflict of interest.

Received: 21 February 2012; accepted: 07 April 2012; published online: 27 April 2012
Citation: Schlaghecken F, Birak KS and Maylor EA (2012) Age-related deficits in efficiency of low-level lateral inhibition. Front. Hum. Neurosci. 6:102. doi: 10.3389/fnhum.2012.00102

Copyright (C) 2012 Schlaghecken, Birak and Maylor. This is an open-access article distributed under the terms of the Creative Commons Attribution Non Commercial License, which permits noncommercial use, distribution, and reproduction in other forums, provided the original authors and source are credited. 